\title{
A Novel OnabotulinumtoxinA Treatment Technique to Obtain Predictable Outcomes in Eyebrow Position and Shape
}

This article was published in the following Dove Press journal:

Clinical, Cosmetic and Investigational Dermatology

\author{
Maria Angelo-Khattar ${ }^{1,2}$ \\ 'American Academy of Anti-Aging \\ Medicine (A4M), Dubai, United Arab \\ Emirates; ${ }^{2}$ Shape Clinic, Kuwait City, \\ Kuwait
}

Background: OnabotulinumtoxinA has been approved for multiple clinical and dermatological indications. The toxin, produced by the bacterium Clostridium botulinum, is undoubtedly the most widely used cosmetic treatment for both the reduction and prophylaxis of wrinkles in the upper face and the reshaping of the eyebrows. However, the aesthetic outcomes obtained with respect to eyebrow position and shape are not always predictable and furthermore, untoward effects such as eyelid and brow ptosis are sometimes seen.

Objective: This article suggests a novel injection technique of the upper face with OnabotulinumtoxinA to obtain predictable and reproducible results with respect to eyebrow position and shape, whilst greatly minimizing the risk of adverse effects such as brow and lid ptosis.

Discussion: OnabotulinumtoxinA inhibits the release of acetylcholine and results in the temporary paralysis of skeletal muscle. The aesthetic outcome of wrinkle reduction typically lasts for a period of 14-16 weeks. Although relatively safe in that the toxin does not have any irreversible side effects, even a temporary untoward reaction may be seriously distressing to the cosmetic patient.

Conclusion: The proposed injection technique, when diligently followed, ensures that the final eyebrow position is pleasing and symmetrical with a negligible risk of any untoward effects.

Keywords: botulinum toxin A, facial wrinkles, brow lift, treatment protocol

\section{Introduction}

OnabotulinumtoxinA, produced by the bacterium Clostridium botulinum inhibits acetylcholine release and hence has a temporary relaxing effect on skeletal muscles. $^{1,2}$ OnabotulinumtoxinA injections for the treatment of hyperdynamic lines and the rebalancing of facial muscles have revolutionized cosmetic practice world-wide. The unparalleled interest in this procedure has brought forth advances in the way in which botulinum toxin can be used to rejuvenate the entire face. ${ }^{3}$ Nonetheless, the forehead and glabella remain the most widely addressed treatment areas with the neuromodulator. ${ }^{4,5}$ Treatment of the brow can achieve a relaxed appearance and may be used to obtain a "chemical brow lift". 4,5

Countless publications have addressed treatment of the upper face with the neurotoxin, describing various dosages and injection points for the reduction of vertical glabellar and horizontal forehead lines. ${ }^{6-9}$
Correspondence: Maria Angelo-Khattar American Academy of Anti-Aging Medicine (A4M), Dubai, United Arab Emirates

Tel +97I506245494

Emailmkhattar@younatagroup.com 
In most areas of the face, levators lift or "elevate" a certain part of the facial anatomy and are opposed by depressors that lower or "depress" that same part of the facial anatomy. In the brow, muscle balance is maintained by the opposition of the only levator, the occipitofrontalis, by the depressor complex including the corrugator supercilii, the procerus, depressor supercilii and the orbicularis oculi. ${ }^{11}$ By treating one or both of the depressors and the levator, it is possible to reduce both glabellar and horizontal forehead dynamic wrinkles, to achieve a smooth brow. However, rebalancing of the levator and depressor brow muscles may result in changes in the position and shape of the eyebrows. Hence although the brow is often regarded as the easiest area of the face to treat with the neuromodulator, the aesthetic results with respect to eyebrow position and shape are not always optimal and reproducible. $^{10}$

The popular press is abounding with the negative effects of over paralyzing the forehead with botulinum toxin, obliterating every line and resulting in a stiff and frozen appearance. The objective of treatment should be to selectively weaken one or both sets of muscles and reduce mimic lines whilst retaining a natural appearance. Hence the patient may remain wrinkle-free at rest but retain a certain amount of movement and expression upon animation. A further goal of rebalancing the brow muscles with the neuromodulator is to obtain an aesthetically pleasing eyebrow position and shape.
This paper describes a simple injection technique to ensure that predictable cosmetic results are obtained each time, with the avoidance of complications such as brow and lid ptosis, as well as unnatural aesthetic outcomes including brow asymmetry, the mephisto sign and residual glabellar and forehead wrinkles.

\section{Ideal Eyebrow Shape}

Figure 1 illustrates ideal eyebrow shape in both genders. In women, the medial eyebrow begins at the same vertical plane as the lateral aspect of the ala and the inner canthus (A). The eyebrow ends laterally at an oblique line drawn from the lateral aspect of the ala through the lateral canthus (B). The medial and lateral aspects of the eyebrow lie at approximately the same horizontal level with the lateral aspect being higher than the medial aspect by 10-20 degrees. The apex lies on a vertical line directly above the lateral limbus (C) such that the ratio of the medial eyebrow to the lateral eyebrow corresponds to the ideal ratio of phi which is $1: 1.618$. In men, the eyebrows are typically thick and bushy, almost invariably straight across, with minimal or no arch. ${ }^{12}$

\section{Materials and Methods}

A retrospective review of 700 patient records was performed for the period between January 2011 and June 2020. The patients had all been treated with

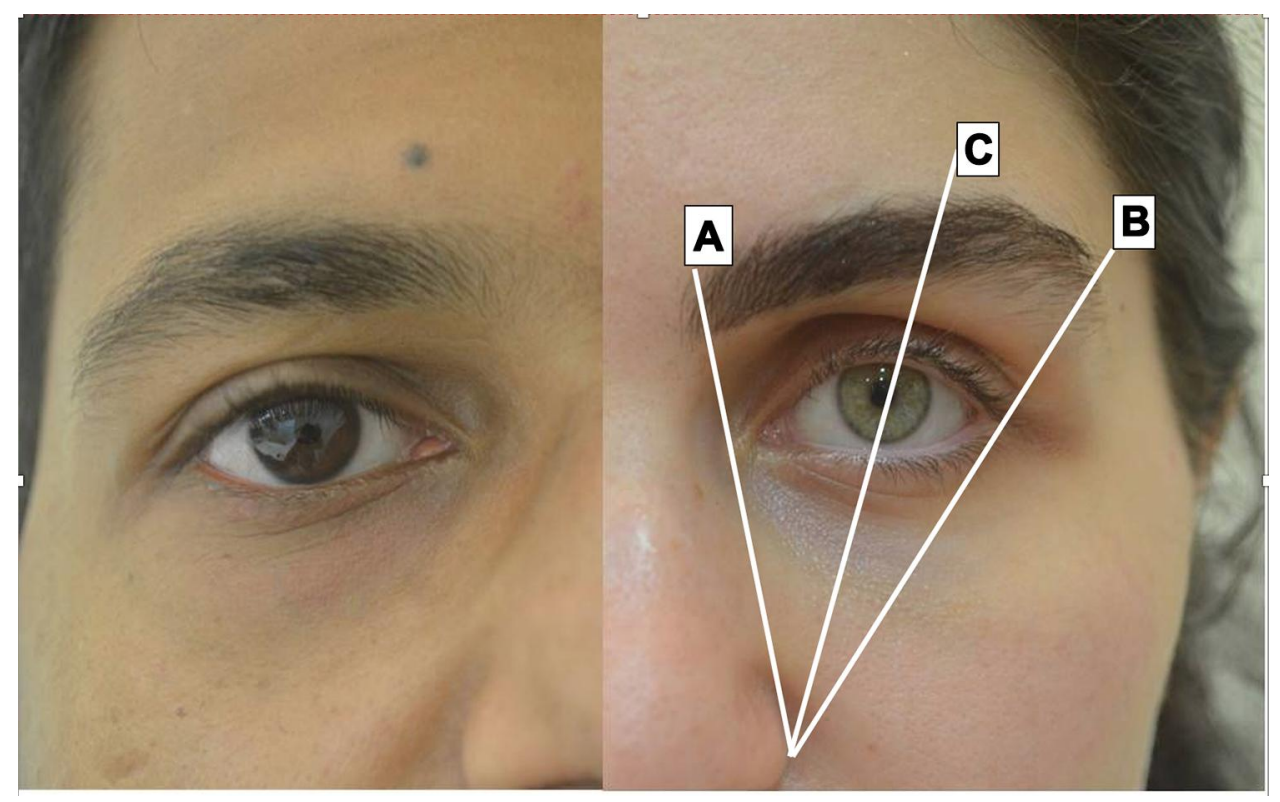

Figure I Ideal eyebrow shapes in men and women.

Notes: Adapted from Yalcinkaya E, Cingi C, Söken H, Ulusoy S, Muluk NB. Aesthetic analysis of the ideal eyebrow shape and position. Eur. Arch. Otorhinolaryngol. 20I6; 273(2):305-3I,0I2). ${ }^{12}$ 
OnabotulinumtoxinA during the training program of the Aesthetic Anti-Aging fellowship conducted by the American Academy of Anti-Aging Medicine (A4M, Dubai, UAE). The brow depressor and levator muscles were treated under the direct supervision of the overseas director of the A4M Aesthetics fellowship (author).

The injection technique outlined in Figure 2 was adopted each time.

Patients presented two weeks after the initial treatment for a follow-up consultation with the director of the training program and the result of the treatment of each patient was documented by obtaining post-treatment standardized photographs (Canfield Vectra 3D Imaging System, Canfield Scientific, New Jersey, USA).

Furthermore, the outcomes were assessed, based upon subjective patient feedback and evaluation by the author and documented on the patient's electronic treatment notes (Capsule, Tendercare, Dubai, UAE). Any patient or physician dissatisfaction with the results was recorded. Safety outcomes of interest including brow and lid ptosis as well as poor aesthetic outcomes such as the development of the mephisto sign, eyebrow asymmetry and the presence of residual glabellar and horizontal forehead lines, were recorded.

\section{Injection Technique \\ The Glabellar Complex}

Is treated with 5 deep perpendicular injections of 4 units each in the Procerus, Depressor supercilii and Corrugator supercilii (Figure 2). The Procerus is identified as the cross-section of two lines drawn from the medial eyebrow on one side to the inner canthus on the contralateral side. The Depressor supercilii and Corrugator supercilii are identified by asking the patient to frown. Typically, in our experience, only one medial point is necessary for each Corrugator supercilii, since in the vast majority of cases, diffusion of the neurotoxin to a radius of $0.5 \mathrm{~cm}$, is sufficient to relax this small muscle. Avoidance of the lateral corrugator skin insertion point, which is usually advocated by many, greatly reduces the risk of lid ptosis.

\section{Forehead Injection Points}

A vertical line is drawn to delineate the mid-line of the forehead (A) and two further vertical lines (B and C) are each drawn at a horizontal distance of $4.5 \mathrm{~cm}$ from the mid-line (Figure 2). This horizontal distance on either side, corresponds approximately to the mid-lateral eyebrow and ensures the equidistant placement from the midline, of the subsequent frontalis neurotoxin injections. Two units of

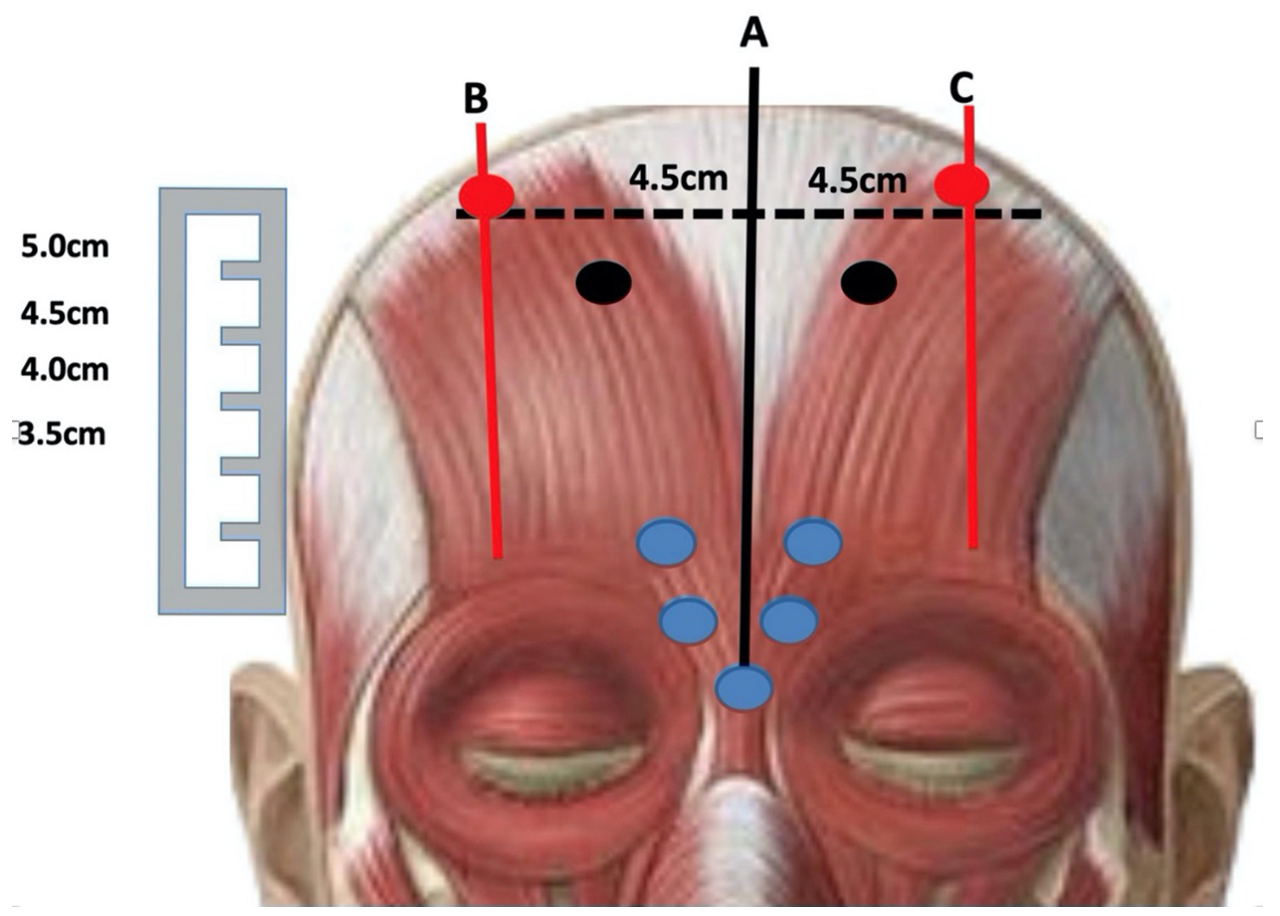

Figure 2 Treatment protocol for eyebrow shaping with OnobotulinumtoxinA. 4 units each in Procerus, Depressor supercilii and Corrugator supercilii. 2 points of 2 units each on Frontalis at measured distances above peak of eyebrows. 2 or more points of 2 units each in upper third to mid-Frontalis for horizontal lines. 


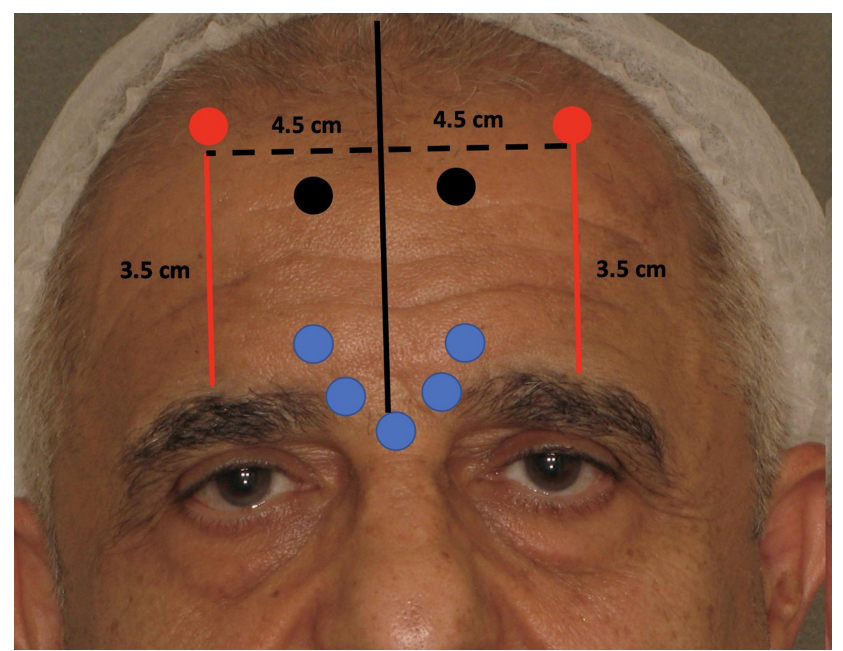

Figure 3 Injection points of OnabotulinumtoxinA on the glabellar complex and frontalis to maintain original eyebrow position. 4 units each in Procerus, Depressors supercilii and Corrugators supercilii. 2 points of 2 units each on Frontalis at $3.5 \mathrm{~cm}$ above the peak of the eyebrows. 2 points of 2 units each in mid-Frontalis for horizontal forehead line.

the neurotoxin are then injected into the frontalis muscle along each of lines $\mathrm{B}$ and $\mathrm{C}$. These injection points are crucial in controlling the position of the eyebrows, avoiding the mephisto sign and ensuring eyebrow symmetry.

Injection points at $3.5 \mathrm{~cm}$ along lines $\mathrm{B}$ and $\mathrm{C}$, starting from the uppermost hairs of the eyebrows, ensure that the eyebrows generally remain in their original position. This is standard protocol in men as the eyebrows should not be allowed to elevate (Figure 3).

Injections of the neurotoxin into the frontalis muscle higher up along lines B and $\mathrm{C}$, at $4.0,4.5$ or $5 \mathrm{~cm}$ (Figure 2) will result in a progressively greater lifting of the eyebrows, respectively (Figure 4). In the event that, during the follow up consultation, the elevation of the eyebrows is deemed to be displeasing to the patient, it is possible to lower the eyebrow position. This may be achieved by injecting 2 point of 2 units each of the neurotoxin, along each of lines $\mathrm{B}$ and $\mathrm{C}$ at 0.5 or $1 \mathrm{~cm}$ lower that the initial injection points.

Horizontal forehead lines are treated by injecting two or more points of 2 units each along the upper one-third to half of the forehead. This ensures a reduction in the wrinkles but does not compromise the position of the eyebrows (Figures 2 and 3).

In cases of pre-existing eyebrow asymmetry where one eyebrow is rather higher than the other, it is possible to correct the asymmetry by lifting the lower eyebrow with a higher injection point and treated the frontalis of the elevated eyebrow at a lower point, typically the difference being 0.5 to $1.0 \mathrm{~cm}$ (Figure 5).

In many cases, once the forehead and glabella are relaxed, compensatory horizontal lines appear on the forehead, just above the lateral eyebrows (Figure 6A).

It is a well-established fact that, in treating forehead rhytids with the neuromodulator, intramuscular injections into the frontalis should be at least $2 \mathrm{~cm}$ above the orbital rim to prevent brow ptosis. However, at this distance from the eyebrows, the neuromodulator cannot often address these bothersome "comma" lines just above the lateral eyebrows (Figure 6A). Hence, in such cases, several superficial intradermal papules of dilute neurotoxin (1.3 units $/ 0.1 \mathrm{~mL}$ ) may be injected, closer to the eyebrows, within the area of these lateral forehead lines. In our experience, this is very effective in mitigating the wrinkles without compromising the elevation of the eyebrows (Figure 6B). It is also possible to pre-empt the development of these "comma" lines

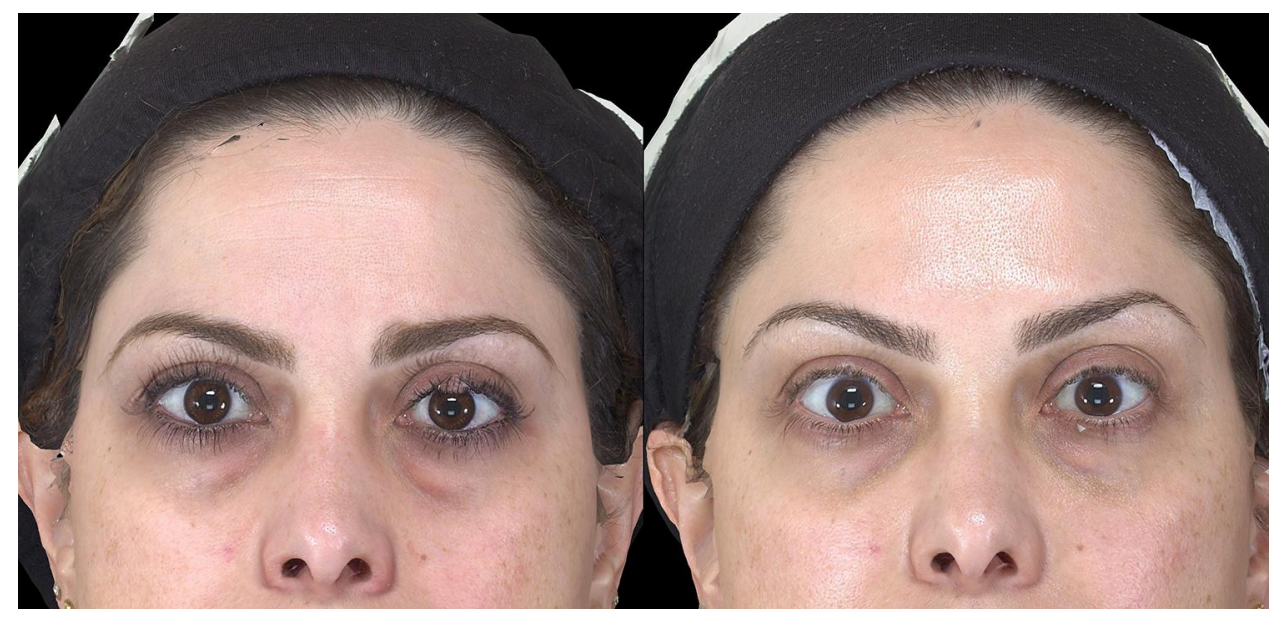

Figure 4 Before and after OnabotulinumtoxinA for eyebrow elevation. 


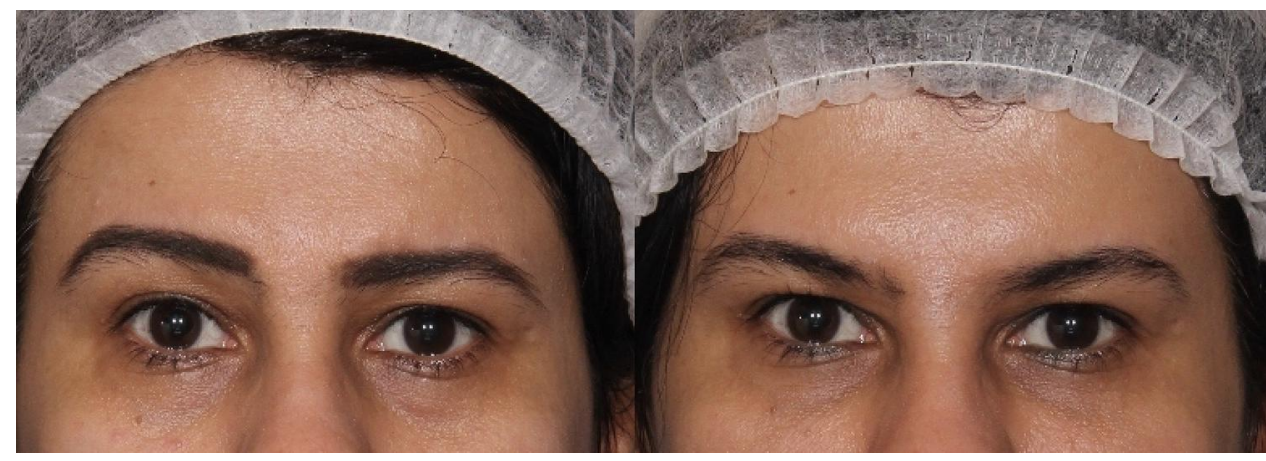

Figure 5 Before and after OnabotulinumtoxinA treatment for the correction of eyebrow asymmetry.

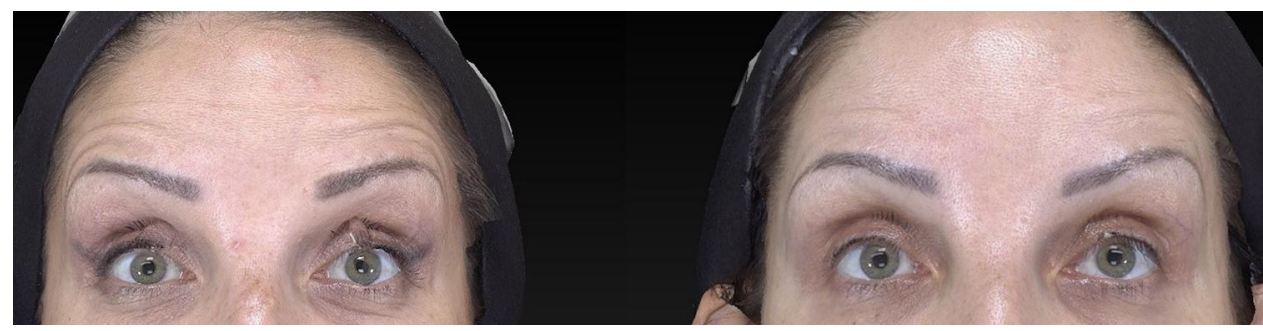

Figure 6 The lateral horizontal forehead lines before $(\mathbf{A})$ and after intradermal injections of dilute OnabotulinumtoxinA (B).

at the initial neurotoxin treatment session by asking the patient to lift their eyebrows and observing whether or not they appear. In such cases, the intradermal microinjections may be performed during the initial treatment session. Alternatively, these lines may be addressed during the follow-up session.

\section{Results}

The retrospective review of the case series of 700 patients showed that both patients and the physician were satisfied with the outcome of the treatment. No incidences of eyelid or brow ptosis were reported by the patients or the physician. The only cosmetic concern in $126(18 \%)$ of the patients, was the presence of a few residual lateral forehead lines that remained posttreatment. These were addressed with a few intradermal papules of the neuromodulator during the follow up consultation.

The results of representative cases, when using the treatment algorithm are shown in Figures 4-7. Written informed consent for publication of the details was obtained from the patient in each case.

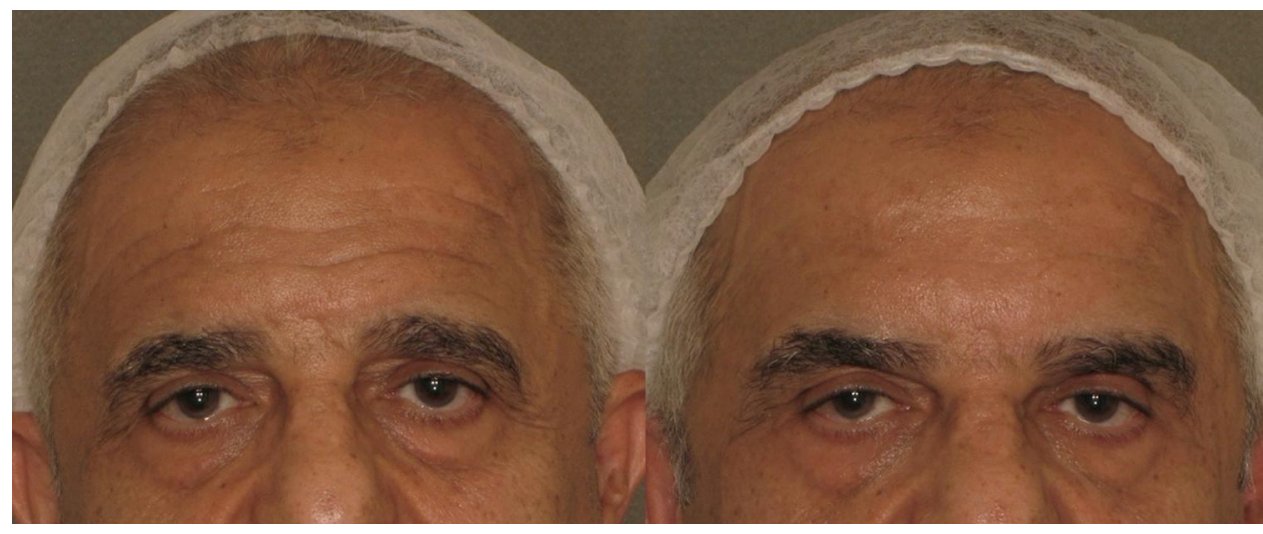

Figure 7 Before and after OnabotulinumtoxinA in a male patient. 
Figure 7 is representative of results in male patients. In this case the frontalis at B and C (Figure 2) was treated with the neuromodulator at $3.5 \mathrm{~cm}$ from the eyebrows to prevent the mephisto sign and unnatural eyebrow elevation. The patient achieved a relaxed appearance with attenuation of the horizontal and vertical lines and retention of his masculine eyebrow shape. The attenuation of the glabellar lines is clearly visible, even though each corrugator supercilii received only one medial dose of 4 units of the neurotoxin and the lateral skin insertion points of the corrugator supercilii was totally avoided.

Figure 4 is a typical result of eyebrow elevation achieved in a female patient, where the frontalis was injected along lines $\mathrm{B}$ and $\mathrm{C}$ at $4.5 \mathrm{~cm}$ above the eyebrows. The patient achieved a relaxed, open-eyed look. The relief of upper eyelid hooding is obvious in this case.

Figure 6 exhibits a common consequence of upper face treatment with botulinum toxin A, namely the development of several "comma" lines just above the eyebrows (Figure 6A). The results of treatment with four intradermal papules of dilute neurotoxin, on each side, are seen as an attenuation of the lines without compromise of the eyebrow position (Figure 6B).

\section{Discussion}

Botulinum toxin A treatment is the cornerstone of minimally invasive facial procedures. It is a well-recognized fact that facial expression and in particular glabellar frown lines and horizontal forehead lines, have an important role in both the patient's self-perception and the perception by others. Botulinum toxin A treatments to the brow depressor and levator muscles have proven to be unique in the reduction and prophylaxis of such wrinkles ${ }^{13}$ as well as in the reshaping and elevation of the eyebrows. However, due to interindividual variations in anatomy, conventional treatment methods with botulinum toxin $\mathrm{A}$ often result in unpredictable outcomes with respect to the eyebrow shape and may also lead to side effects such as lid and brow ptosis. ${ }^{14}$ This retrospective case series of 700 patients has shown that adopting the proposed technique, based on standardized measurements, allowed us to consistently obtain positive aesthetic outcomes in terms of reduction of both glabellar as well as horizontal forehead lines whilst retaining a symmetrical and pleasing eyebrow shape. Moreover, in all of the cases treated, neither lid nor brow ptosis was observed. The absence of brow ptosis was due to the fact that the frontalis was not over-injected, and the lowest point treated on the forehead, was at $3.5 \mathrm{~cm}$ from the eyebrows. Moreover, the absence of lid ptosis was probably due to the fact that the corrugator supercilii were only injected medially and that the lateral skin insertion point was avoided. Hence the possibility of diffusion of the toxin to the levator palpebrae superioris with resultant lid ptosis, was eliminated.

\section{Conclusion}

Botulinum toxin A is an invaluable tool in our arsenal of aesthetic treatments. It is deemed to be a relatively safe and simple treatment, nonetheless, cosmetic results with respect to eyebrow position and shape are not always optimal. Furthermore, the treatment carries a risk of adverse effects such as brow and lid ptosis.

A novel technique based on specific measurements was used to treat 700 patients. The retrospective review of the cases showed positive aesthetic outcomes with respect to eyebrow position and shape and more importantly, there were no incidences of brow or lid ptosis.

The proposed treatment protocol offers the aesthetic practitioner a means of consistently ensuring optimal cosmetic treatment outcomes with a very low risk of complications. Finally, diligent documentation of the protocol adopted for each individual patient, allows for reproducible results upon subsequent treatments.

Research is ongoing to obtain a validated treatment algorithm.

\section{Acknowledgments}

I wish to thank the patients who have given me permission to publish their photos.

I also wish to thank Dr. Ahmed Yasin for his advice.

\section{Disclosure}

The author reports no conflicts of interest in this work.

\section{References}

1. Trindade de Almeida AR, Secco LC, Carruthers A. Handling botulinum toxins: an updated literature review. Dermatol Surg. 2011;37 (11):1553-1565. doi:10.1111/j.1524-4725.2011.02087.x

2. Samizadeh S, De Boulle K. Botulinum neurotoxin formulations: overcoming the confusion. Clin Cosmet Investig Dermatol. 2018;11:273-287. doi:10.2147/CCID.S156851

3. Carruthers J, Carruthers A. The evolution of botulinum neurotoxin type A for cosmetic applications. J Cosmet Laser Ther. 2007;9 (3):186-192. doi:10.1080/14764170701411470

4. Benedetto AV. Cosmetic uses of botulinum toxin A in the upper face. In: Benedetto AV, editor. Botulinum Toxin in Clinical Dermatology. Taylor and Francis; 2006:50-152.

5. De Boulle K, Carruthers S, Solish N, et al. OnabotulinumtoxinA treatment for moderate to severe forehead lines: a review. Plast Reconstr Surg Glob Open. 2020;8(3):e2669. doi:10.1097/ GOX.0000000000002669

6. Small R. Botulinum toxin injection for facial wrinkles. Am Fam Physician. 2014;90(3):168-175. 
7. Kwolek MS, Block JE. Maximizing botulinum toxin injections for cosmetic and therapeutic applications with a single use, disposable exact dose injection assist device. Clin Cosmet Investig Dermatol. 2019;12:35-46. doi:10.2147/CCID.S189595

8. Jun J-Y, Park J-H, Youn CS, Lee JH. Jong hee lee intradermal injection of botulinum toxin: a safer treatment modality for forehead wrinkles. Ann Dermatol. 2018;30(4):458-461. doi:10.5021/ad.2018.30.4.458

9. Framptom JE, Eathope SE. Botulinum toxin A (Botox cosmetic): a review of its use in glabellar frown lines. Am J Clin Dermatol. 2003;4(10):709-725. doi:10.2165/00128071-200304100-00005

10. Gendler E, Nagler A. Aesthetic use of BoNT: options and outcomes. Toxicon. 2015;107(Pt A):120-128. doi:10.1016/j.toxicon.2015.08.023

11. Abramo AC, Do Amaral TP, Lessio BP, De Lima GA. Anatomy of forehead, glabellar, nasal and orbital muscles, and their correlation with distinctive patterns of skin lines on the upper third of the face: reviewing concepts. Aesthetic Plast Surg. 2016;40(6):962-971. doi:10.1007/s00266-016-0712-z
12. Yalcinkaya E, Cingi C, Söken H, Ulusoy S, Muluk NB. Aesthetic analysis of the ideal eyebrow shape and position. Eur Arch Otorhinolaryngol. 2016;273(2):305-310. doi:10.1007/s00405-014-3356-0

13. Binder WJ. Long-term effects of botulinum toxin type A (Botox) on facial lines. Arch Facial Plast Surg. 2006;8(6):426-431. doi:10.1001/ archfaci.8.6.426

14. Sundaram H, Signorini M, Liew S, et al. Global aesthetics consensus group. Global aesthetics consensus: botulinum toxin type $\mathrm{A}-$ evidence-based review, emerging concepts, and consensus recommendations for aesthetic use, including updates on complications. PRS. 2016;137(3):518e-529e

\section{Publish your work in this journal}

Clinical, Cosmetic and Investigational Dermatology is an international, peer-reviewed, open access, online journal that focuses on the latest clinical and experimental research in all aspects of skin disease and cosmetic interventions. This journal is indexed on CAS.
The manuscript management system is completely online and includes a very quick and fair peer-review system, which is all easy to use. Visit http://www.dovepress.com/testimonials.php to read real quotes from published authors. 